TITLE:

\title{
Size-selective extended X-ray absorption fine structure spectroscopy of free selenium clusters
}

\section{AUTHOR(S):}

Nagaya, K; Yao, M; Hayakawa, T; Ohmasa, Y; Kajihara, Y; Ishii, M; Katayama, Y

\section{CITATION:}

Nagaya, K ... [et al]. Size-selective extended X-ray absorption fine structure spectroscopy of free selenium clusters. PHYSICAL REVIEW LETTERS 2002, 89(24): 243401.

\section{ISSUE DATE:}

2002-12-09

URL:

http://hdl.handle.net/2433/50034

RIGHT:

Copyright 2002 American Physical Society 


\title{
Size-Selective Extended X-ray Absorption Fine Structure Spectroscopy of Free Selenium Clusters
}

\author{
K. Nagaya, M. Yao, ${ }^{*}$ T. Hayakawa ${ }^{\dagger}$ Y. Ohmasa, and Y. Kajihara \\ Department of Physics, Graduate School of Science, Kyoto University, Sakyo-ku, Kyoto 606-8502, Japan \\ M. Ishii \\ SPring-8/JASRI, Mikazuki, Sayo, Hyogo 679-5148, Japan \\ Y. Katayama \\ SPring-8/JAERI, Mikazuki, Sayo, Hyogo 679-5148, Japan \\ (Received 8 April 2002; published 22 November 2002)
}

\begin{abstract}
In a recent paper [M. Yao et al., J. Synchrotron Radiat. 8, 542 (2001)], we proposed a new method for the size-selective EXAFS (extended x-ray absorption fine structure) of neutral-free clusters, in which not only the $\mathrm{x}$-ray absorption process but also the deexcitation processes are utilized as the structural information. In order to verify this method experimentally, we have developed the synchronous measurements of EXAFS and photoelectron photoion coincidence and carried them out for a Se cluster beam by utilizing the third-generation intense $\mathrm{x}$-ray source. The EXAFS spectra for Se small clusters have been obtained and compared critically with theoretical predictions.
\end{abstract}

DOI: $10.1103 /$ PhysRevLett.89.243401

PACS numbers: $36.40 . \mathrm{Mr}, 61.10 . \mathrm{Ht}$

Although free clusters attract great interest as the intermediate states between atomic and condensed states of matter [1], general methods for the size-selective structural analysis of neutral clusters have not been established thus far. The electron diffraction has been successfully applied to relatively large clusters [2] which exhibit the Bragg peaks due to the long range order, but it becomes less useful as the cluster size decreases. In this respect, EXAFS (extended x-ray absorption fine structure) spectroscopy is rather promising [3-6] because the short range order dominates the spectrum. However, the absorption of the $\mathrm{x}$-ray photon induces massive fragmentation [3,7], and the problem is extremely serious when the deep inner-core levels are excited, which conflicts with a general view that the EXAFS spectrum is more reliable when the core hole is more localized. Furthermore, in these conventional methods, the sizespecific information can be deduced only by combining many experimental runs with various stagnation conditions $[2-6,8]$.

Recently, we measured PEPICO (photoelectron photoion coincidence) spectra of selenium dimer $\mathrm{Se}_{2}$ and larger species such as $\mathrm{Se}_{5}[7,9]$. The PEPICO spectra reveal that multiply charged ions are produced as a result of deexcitation processes following the $K$ shell excitation and fragment into atomic ions with $z$ charges, $\mathrm{Se}^{z+}$, owing to the Coulomb explosion [10]. The branching ratios from the parent neutral cluster to the daughter ions exhibit clear dependence on the size $n$ of the parent cluster. In particular, the branching ratios in the nonresonant absorption region are well reproduced by considering that the charges are randomly distributed within the cluster before the Coulomb explosion. Based upon these findings, we proposed a new method for the size-selective EXAFS of neutral-free clusters by utilizing the PEPICO measurements [11].

The basic relations we have suggested are the simultaneous equations of the x-ray absorption coefficients per atom, $\alpha(n, h \nu)$, parametrized by $z$, as follows:

$$
P(z, h \nu)=\sum_{n} A(n) B(n, z) \alpha(n, h \nu) .
$$

Here $P(z, h \nu)$ is the partial ion yield (PIY) of the $z$ charged ion, deduced from the integrated PEPICO intensity. $A(n)$ is the abundance of parent clusters consisting of $n$ atoms and $B(n, z)$ is the branching ratio. Since $A(n)$ and $B(n, z)$ can be estimated either from experiments or calculations, $\alpha(n, h \nu)$ could be deduced by solving Eq. (1) from the PEPICO measurements. A crucial advantage of this method is that the size-selective information could be acquired, in principle, in a single experimental run.

The aim of the present work is to verify Eq. (1) experimentally. For this purpose, we have developed the EXAFS-PEPICO synchronous measurements and carried them out for the Se cluster beam at the $\mathrm{X}$-ray undulator beam line BL10XU installed in SPring-8 [12]. In this Letter, we will describe the procedure to deduce the sizeselective EXAFS oscillation $\chi_{n}(k)$ and compare the structural parameters estimated from the $\chi_{n}(k)$ with several theoretical calculations.

The neutral cluster beam was produced by a supersonic jet expansion method [7], intersected with the x-ray at right angles in the horizontal. The photoions produced by absorbing an $\mathrm{x}$-ray photon were extracted upward by a constant electric field to a detector (channeltron), while the photoelectrons were extracted downward. The 
EXAFS spectrum for the whole cluster beam [i.e., nonsize-selective $\chi_{T}(k)$ ] was taken in the total ion yield (TIY) mode. At each photon energy where the TIY was measured, the PEPICO spectrum was recorded simultaneously. The photoelectron signal delivers the start pulse and the photoion signal delivers the stop pulse for a timeamplitude converter, and the difference of the time of flight between the electron and ion was accumulated as a histogram in a multichannel analyzer (MCA). We have modified the software of the beam line host computer, which otherwise serves to measure only the EXAFS spectrum, so that it can also control the MCA to measure the PEPICO spectrum synchronously [13].

As in the previous works [7], $\mathrm{Se}_{2}, \mathrm{Se}_{5}, \mathrm{Se}_{6}$, and $\mathrm{Se}_{7}$ were included in the cluster beam produced in our small cluster apparatus. In the present study we kept the temperature of the cluster source at $703 \mathrm{~K}$ and the relative abundance [i.e., $A(n)$ in Eq. (1)] of these clusters was 46:27:18:9, which was preliminarily studied mass spectrometrically and confirmed during the $\mathrm{x}$-ray absorption measurements by the amount of jump in $P(z, h \nu)$ at the $K$ edge. Further experimental details will be described elsewhere [13].

Figure 1 shows the total ion yield (TIY) spectrum versus X-ray photon energy for the Se cluster beam. Although the number density $\left(3.5 \times 10^{11} \mathrm{~cm}^{-3}\right)$ of $\mathrm{Se}$ atoms in the cluster beam is smaller than that of typical bulk samples by more than 10 orders of magnitude, the EXAFS oscillation is clearly seen. The EXAFS spectrum $\chi_{T}(k)$ deduced from the TIY spectrum is displayed in the inset as a function of the photoelectron wave number $k$. Here we have adopted a normal procedure to extract $\chi_{T}(k)$ : The background was subtracted by using a

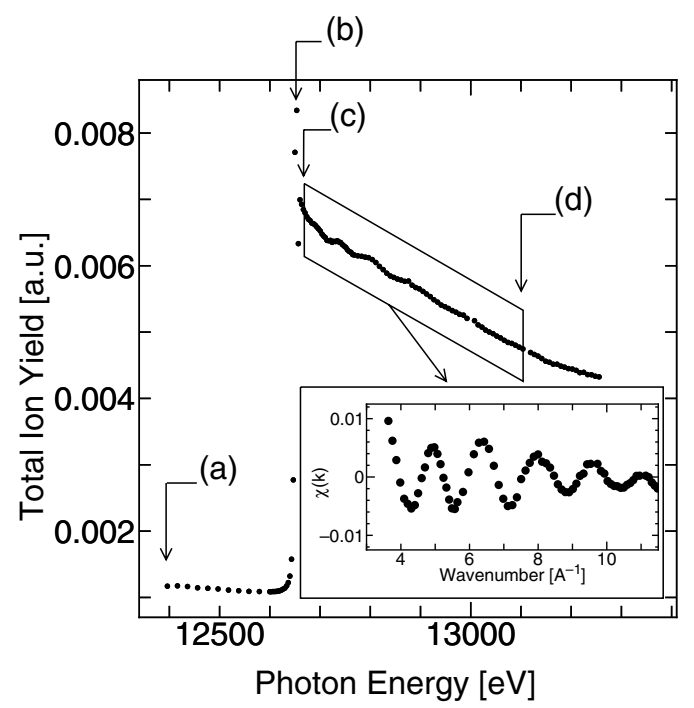

FIG. 1. The total ion yield (TIY) versus $\mathrm{x}$-ray photon energy for the Se cluster beam. Unusual data points due to the Bragg reflection from the monochromator crystal were omitted. The inset shows the EXAFS spectrum $\chi_{T}(k)$ deduced from the TIY as a function of the photoelectron wave number.
Victooren function and the base line of the EXAFS oscillation was expressed by a spline function [14].

Representative PEPICO spectra are shown in Fig. 2. The peaks corresponding to $z=1$ to 7 are observed. The asymmetrical splitting is clear evidence for the Coulomb explosion [7]. The spectrum 2(a) was recorded at the preedge region, 2(b) at the $\sigma^{*}$ resonant energy, 2(c) at the beginning of the EXAFS spectrum, and 2(d) at its end. The arrows in Fig. 1 correspond to these energies. In the EXAFS region the $z=2$ peak is the most pronounced and the $z=1$ is the second largest, and the relative intensity of each peaks changes little with the photon energy. In this region the neutral clusters can be directly ionized by absorbing the $\mathrm{x}$-ray photon, and the generated core-hole states are completely relaxed before the dissociation [9]. For the $\sigma^{*}$ resonance, on the other hand, the $z=1$ peak is appreciably enhanced, implying that the fast dissociation [15] could partially take place [9].

Following the same procedure as adopted when $\chi_{T}(k)$ was deduced from the TIY spectrum, one can extract the partial EXAFS spectrum $\chi_{z}(k)$ for each $z$ from the PIY spectra. In Fig. $3, \chi_{z}(k)$ are displayed for $z=1$ to 7 . The statistical uncertainty is indicated by the error bars near $k=3.8 \AA^{-1}$. There are still clear oscillations in the spectra except for $z=6$ and 7 . For $z=1$ and 2, the spacing of the oscillation is nearly the same. The maxima of the oscillations are marked by the closed squares. For $z=3$, however, the oscillation is disturbed and new peaks denoted by the open squares appear at the high $k$ region. The new series of oscillation prevails for $z=4$ and 5 . The spacing of oscillation seems to be large for large $z$, suggesting that the daughter ions with large $z$ stem mainly from $\mathrm{Se}_{2}$ with short bond length. This is fully consistent with our previous observation on the branching ratio [7] (see Table I). Thus qualitatively the experimental results provide a firm basis to Eq. (1).

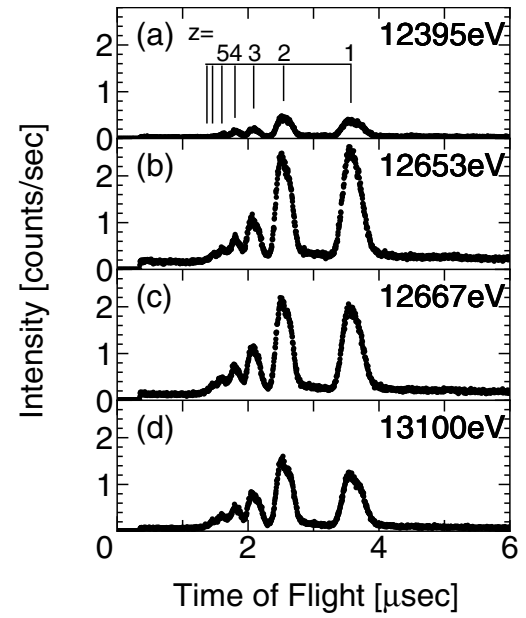

FIG. 2. The PEPICO spectrum versus time of flight for the Se cluster beam at the preedge region (a), at the resonance absorption region (b), at the beginning (c), and end (d) of the EXAFS region. 


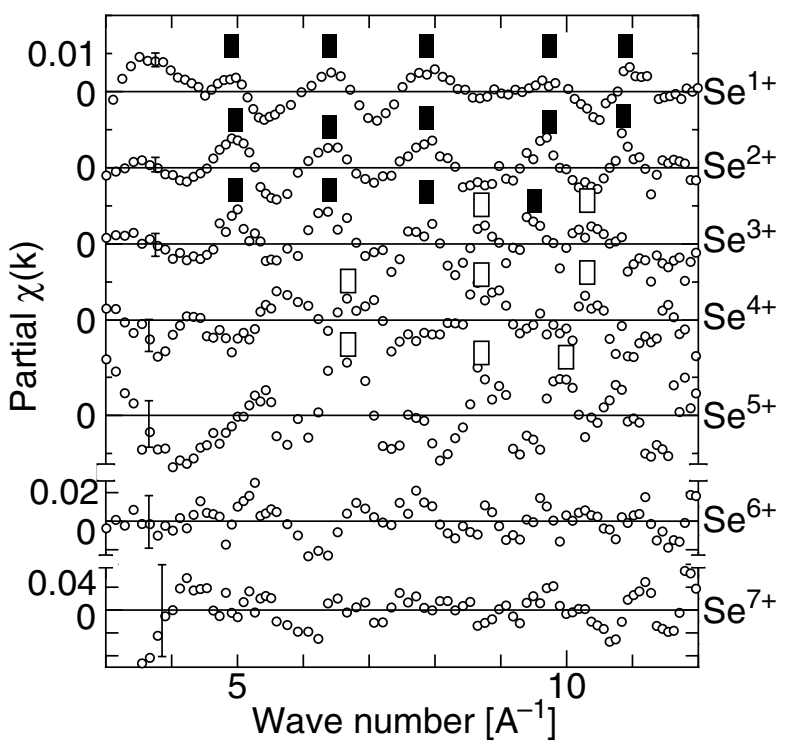

FIG. 3. The partial EXAFS spectrum $\chi_{z}(k)$ for $z=1$ to 7 deduced from the partial ion yields.

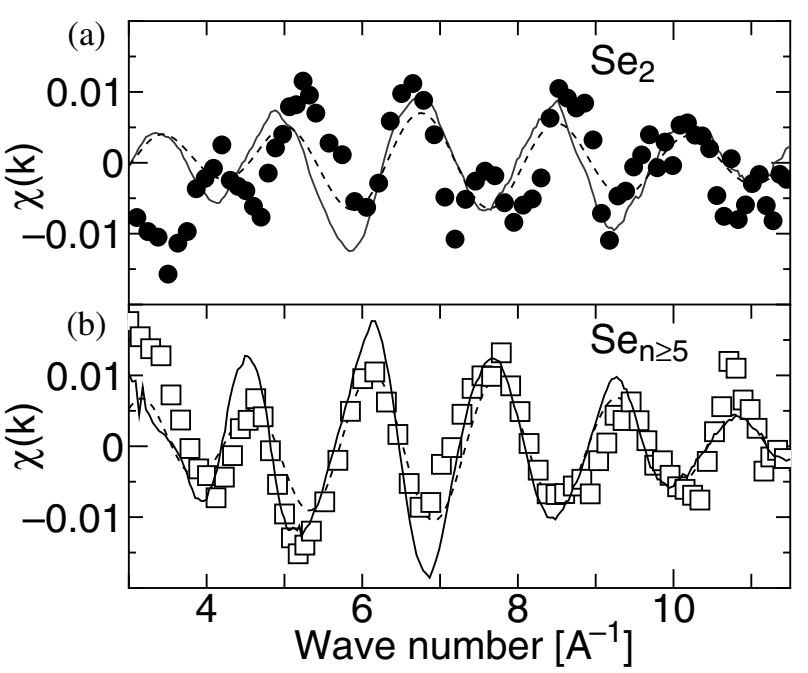

FIG. 4. Size-selective EXAFS spectra $\chi(k)$ for $\mathrm{Se}_{2}$ [circles in (a)] and $\mathrm{Se}_{n \geq 5}$ [squares in (b)]. $\chi(k)$ for $\mathrm{v}$-Se and $\mathrm{m}$-Se are shown by the lines in (a) and (b), respectively. The dashed lines are the filtered Fourier transform of the first peak.

Now let us extract the size-selective EXAFS spectra. To make the problem more tractable, we simplify Eq. (1) as follows:

$$
\left(\begin{array}{c}
\sum_{z=1}^{3} P(z, h \nu) \\
\sum_{z=4}^{7} P(z, h \nu)
\end{array}\right)=\left(\begin{array}{cc}
A(2) \sum_{z=1}^{3} B(2, z) & \sum_{z=1}^{3} \sum_{n=5}^{7} A(n) B(n, z) \\
A(2) \sum_{z=4}^{7} B(2, z) & \sum_{z=4}^{7} \sum_{n=5}^{7} A(n) B(n, z)
\end{array}\right)\left(\begin{array}{c}
\alpha(n=2, h \nu) \\
\alpha(n \geq 5, h \nu)
\end{array}\right) .
$$

Here we have bracketed $\mathrm{Se}_{5}, \mathrm{Se}_{6}$, and $\mathrm{Se}_{7}$ together in $\mathrm{Se}_{n \geq 5}$. We have divided the range of $z$ into two, $(1,2,3)$ and $(4,5,6,7)$.

The resultant $\chi_{n=2}(k)$ for $\mathrm{Se}_{2}$ and $\chi_{n \geq 5}(k)$ for $\mathrm{Se}_{n \geq 5}$ are plotted in Figs. 4(a) and 4(b), respectively. The present $\chi_{n=2}(k)$ is in good agreement with $\chi(k)$ for selenium vapor (v-Se) [16] in the high $k$ region, whereas $\chi_{n \geq 5}(k)$ has the oscillation similar to $\chi(k)$ for monoclinic $\mathrm{Se}$ (m-Se) composed of $\mathrm{Se}_{8}$ [17]. The dashed lines denote the Fourier filtered first-shell signal. By using the FEFF fitting program [18], the bond length $R_{1}$, the DebyeWaller factor $\sigma_{\mathrm{DW}}$, and the scaling factor times coordination number, $S \times N_{1}$, have been estimated and listed in Table II. It is noted that the ratio of $S \times N_{1}$ for $\mathrm{Se}_{2}$ and $\mathrm{Se}_{n \geq 5}$ is nearly 1:2, suggesting that the latter has the ring structure.

Hohl et al. [22] studied the equilibrium geometries of selenium clusters by using ab initio molecular dynamics simulations and predicted that $\mathrm{Se}_{5}, \mathrm{Se}_{6}$, and $\mathrm{Se}_{7}$ have the $\mathrm{C}_{\mathrm{s}}, \mathrm{D}_{3 \mathrm{~d}}$, and $\mathrm{C}_{\mathrm{s}}$ chair structure, respectively. The symmetry tells that there exists one bond length in $\mathrm{Se}_{6}$, three different bond lengths in $\mathrm{Se}_{5}$, and four different ones in

TABLE I. Branching ratios in $\%$ for $\mathrm{Se}_{2}$ and $\mathrm{Se}_{n \geq 5}$ [7].

\begin{tabular}{cccccccc}
\hline \hline & $z=1$ & 2 & 3 & 4 & 5 & 6 & 7 \\
\hline $\mathrm{Se}_{2}$ & 16.6 & 26.6 & 26.7 & 18.0 & 8.6 & 2.9 & 0.6 \\
$\mathrm{Se}_{n \geq 5}$ & 59.9 & 29.3 & 8.8 & 1.8 & 0.2 & 0.02 & 0.001 \\
\hline \hline
\end{tabular}

$\mathrm{Se}_{7}$. They also reported the structural parameters such as bond lengths, bond angles, and torsion angles. Subsequent theoretical works $[23,21,24]$ confirmed that the most favorable symmetries of the clusters are the same as those suggested by Hohl et al. [22]. Experimentally, Becker et al. [25] recorded the valence electron spectra of the Se clusters and revised the structural parameters by combining their data with the semiempirical Hartree-Fock calculations. Making use of these theoretical values, one can estimate the average bond length $R_{1}$ that can be compared with the present experiment, where $\mathrm{Se}_{5}, \mathrm{Se}_{6}$, and $\mathrm{Se}_{7}$ exist with the ratio of 3:2:1. The distribution of

TABLE II. The bond length $\left(R_{1}\right)$, scaling factor times coordination number $\left(S \times N_{1}\right)$, and Debye-Waller factor $\left(\sigma_{\mathrm{DW}}\right)$ derived from the size-selective EXAFS spectra. Vibrational contribution $\sigma_{\text {vib }}$ is estimated from Eq. (4). The static dispersion $\sigma_{\text {stat }}$ is derived from Eq. (3). Two different $\nu_{\text {bs }}$ are used to estimate $\sigma_{\mathrm{vib}}$. Numbers in parentheses denote an error.

\begin{tabular}{cccccc}
\hline \hline & $R_{1}[\AA]$ & $S \times N_{1}$ & $\sigma_{\text {DW }}[\AA]$ & $\sigma_{\text {vib }}[\AA]$ & $\sigma_{\text {stat }}[\AA]$ \\
\hline $\mathrm{Se}_{2}$ & $2.17(1)$ & $0.34(5)$ & $0.032(4)$ & $0.033(0)$ & $\sim 0$ \\
& & & & $0.0415(3)^{\mathrm{a}}$ & $0.016(5)^{\mathrm{a}}$ \\
$\mathrm{Se}_{n \geq 5}$ & $2.35(1)$ & $0.72(2)$ & $0.045(2)$ & & \\
& & & & $0.0395(0)^{\mathrm{b}}$ & $0.021(4)^{\mathrm{b}}$ \\
\hline \hline
\end{tabular}

${ }^{\mathrm{a}}$ Ref. [19,20]. ${ }^{\mathrm{b}}$ Ref. [21]. 
TABLE III. Comparison of $R_{1}$ and $\sigma_{\text {stat }}$ between experimental and theoretical values for $\mathrm{Se}_{n \geq 5}$.

\begin{tabular}{cccccc}
\hline \hline & Present work & Becker $^{\mathrm{a}}$ & Hohl $^{\mathrm{b}}$ & Kohara $^{\mathrm{c}}$ & Pan $^{\mathrm{d}}$ \\
\hline$R_{1}[\AA]$ & $2.35(1)$ & 2.349 & 2.349 & 2.327 & 2.452 \\
& $0.016(5)^{\mathrm{e}}$ & & & & \\
$\sigma_{\text {stat }}[\AA]$ & & 0.019 & 0.057 & 0.026 & 0.086 \\
& $0.021(4)^{\mathrm{c}}$ & & & & \\
\hline \hline
\end{tabular}

${ }^{\mathrm{a}}$ Ref. [25]. ${ }^{\mathrm{b}}$ Ref. [22]. ${ }^{\mathrm{c}}$ Ref. [21]. ${ }^{\mathrm{d}}$ Ref. [24]. ${ }^{\mathrm{e}}$ Refs. [19,20].

the bond lengths can be taken as the static contribution $\sigma_{\text {stat }}$ to the Debye-Waller factor. Both $R_{1}$ and $\sigma_{\text {stat }}$ are listed in Table III for $\mathrm{Se}_{n \geq 5}$. The value of $R_{1}, 2.349 \AA$, predicted by both Hohl et al. [22] and Becker et al. [25] is in very good agreement with our experimental value.

In order to compare the theoretical $\sigma_{\text {stat }}$ with our experimental $\sigma_{\mathrm{DW}}$, we have to subtract the dynamic contribution $\sigma_{\text {vib }}$ due to the molecular vibration by using [26]

$$
\sigma_{\mathrm{DW}}^{2}=\sigma_{\text {stat }}^{2}+\sigma_{\mathrm{vib}}^{2} \text {. }
$$

Here $\sigma_{\text {vib }}(T)$ can be calculated from [26]

$$
\sigma_{\mathrm{vib}}^{2}=\frac{h}{4 \pi^{2} m \nu_{\mathrm{bs}}} \operatorname{coth} \frac{h \nu_{\mathrm{bs}}}{2 k_{\mathrm{B}} T},
$$

where $m$ is the atomic mass and $\nu_{\mathrm{bs}}$ is the bond-stretching frequency estimated from the Raman scattering.

First, we estimate the experimental $\sigma_{\text {stat }}$ for $\mathrm{Se}_{2}$. The temperature of the cluster beam is estimated to be around 80 K [13] by using Hagena's scaling law [27]. Putting an experimental value of $385 \mathrm{~cm}^{-1}$ [28] to $\nu_{\mathrm{bs}}$, we obtain $0.033 \AA$ as $\sigma_{\text {vib }}$, which is dominated by the zero-point motion. Thus, it proves that $\sigma_{\text {stat }}$ is nearly zero for $\mathrm{Se}_{2}$ in accordance with the fact that there exists only one species for $\mathrm{Se}_{2}$.

Similarly, $\sigma_{\text {vib }}$ can be estimated for $\mathrm{Se}_{n \geq 5} . \nu_{\mathrm{bs}}$ is about $250 \mathrm{~cm}^{-1}$ for both orthorhombic $\left(\mathrm{Se}_{7}\right)$ [19] and rhombohedral $\left(\mathrm{Se}_{6}\right)$ [20] crystalline forms of selenium. On the other hand, Kohara [21] proposed $276 \mathrm{~cm}^{-1}$ as $\nu_{\mathrm{bs}}$ for $\mathrm{Se}_{6}$ based upon the $a b$ initio molecular orbital calculation. Table II contains $\sigma_{\text {vib }}$ and $\sigma_{\text {stat }}$ estimated from these $\nu_{\text {bs }}$ values. Again Becker's result agrees with our experimental value (see Table III).

In conclusion, we have verified the size-selective EXAFS method for neutral-free clusters by performing the EXAFS-PEPICO synchronous measurements on a selenium cluster beam. The quantitative comparison of the average bond length and the Debye-Waller factor between the present experiment and several previous works suggests that the prediction by Becker et al. [25] seems to be the best. We believe that this method is general as far as the clusters are small and composed of heavy elements, where the core holes are completely relaxed before the Coulomb explosion [9].
This work was collaborated with Mr. Y. Nakagawa and K. Hamada. This work was partially supported by a grant-in-aid for Scientific Research from the MEXT, Japan.

*Corresponding author.

Electronic address: yao@scphys.kyoto-u.ac.jp

†Present address: Genesis Research Institute, Inc., Ichikawa 272-0001, Japan.

[1] Clusters of Atoms and Molecules, edited by H. Haberland (Springer, New York, 1994).

[2] B. Raoult et al., Rev. Sci. Instrum. 44, 430 (1973); G. Torchet et al., J. Chem. Phys. 103, 3074 (1995); J. Farges et al., J. Chem. Phys. 78, 5067 (1983); D. Reinhard et al., Phys. Rev. Lett. 79, 1459 (1997); D. Reinhard et al., Phys. Rev. B 55, 7868 (1997); B.W. van de Waal, Z. Phys. D 20, 349 (1991).

[3] E. Rühl, J. Chem. Phys. 98, 2653 (1993); E. Rühl et al., J. Chem. Phys. 98, 6820 (1993).

[4] S. Kakar et al., Phys. Rev. Lett. 78, 1675 (1997).

[5] F. Federmann et al., Phys. Rev. Lett. 73, 1549 (1994).

[6] C. M. Teodorescu et al., J. Chem. Phys. 109, 9280 (1998).

[7] T. Hayakawa et al., J. Phys. Soc. Jpn. 69, 2039 (2000).

[8] M. Yao et al., Jpn. J. Appl. Phys. Suppl. 38-1, 38, 564 (1999).

[9] T. Hayakawa et al., J. Phys. Soc. Jpn. 69, 2850 (2000).

[10] O. Echt et al., Phys. Rev. A 38, 3236 (1988).

[11] M. Yao et al., J. Synchrotron Radiat. 8, 542 (2001).

[12] H. Oyanagi et al., J. Synchrotron Radiat. 6, 155 (1999).

[13] K. Nagaya, J. Phys. Soc. Jpn. (to be published).

[14] X-ray Absorption: Principles, Applications, Techniques of EXAFS, SEXAFS and XANES, edited by D.C. Koningsberger and R. Prins (Wiley, New York, 1988), Chap. 6.

[15] P. Morin et al., Phys. Scr. T17, 171 (1987).

[16] S. Hosokawa et al., J. Chem. Phys. 97, 786 (1992).

[17] M. Inui et al., J. Phys. Soc. Jpn. 57, 553 (1988); H. Endo et al., Jpn. J. Appl. Phys. 32, Suppl. 32-2, 773 (1993).

[18] A. L. Ankudinov et al., Phys. Rev. B 58, 7565 (1998).

[19] K. Nagata et al., Jpn. J. Appl. Phys. 23, 704 (1984).

[20] K. Nagata et al., Jpn. J. Appl. Phys. 20, 463 (1981).

[21] S. Kohara et al., Chem. Phys. Lett. 287, 282 (1998).

[22] D. Hohl et al., Chem. Phys. Lett. 139, 540 (1987).

[23] G. Igel-Mann et al., Mol. Phys. 80, 341 (1993).

[24] B. C. Pan et al., Phys. Rev. B 62, 17026 (2000).

[25] J. Becker et al., Z. Naturforsch. A 46, 453 (1991).

[26] EXAFS: Basic Principles and Data Analysis, edited by B. K. Teo (Springer, New York, 1986), Chap. 5; Molecular Vibrations and Mean Square Amplitudes, edited by S. J. Cyvin (Elsevier, New York, 1968), Chap. 5.

[27] O. F. Hagena, Z. Phys. D 4, 291 (1987).

[28] Molecular Spectra and Molecular Structure IV, edited by K. P. Huber and G. Herzberg (Van Nostrand Reinhold, New York, 1979), p. 582. 